Military Technical College

Kobry El-Kobba, Cairo, Egypt.

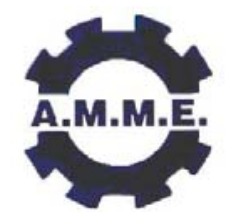

$13^{\text {th }}$ International Conference on Applied Mechanics and Mechanical Engineering.

\title{
ANALYSIS OF LAMINATED CYLINDRICAL PRESSURE VESSELS WITH PRE-LOADS
}

\author{
MOHAMED* E.A.
}

\begin{abstract}
A new closed form analytical solution for laminated cylindrical pressure vessels with lateral pressure is presented. The solution includes nonlinear effects due to large deformations based on Timoshenko approach. The solution also includes the effects of pre-stress which is encountered during the filament winding of composite pressure vessels. The solution allows the analysis of symmetric and asymmetric stacking sequences.

The derivation of the governing equations and its solution is presented. Special case studies for the solution are discussed. Some examples are presented for two different stacking sequences; the effects of length, loading pressure, and pre-stress are studied.
\end{abstract}

\section{KEYWORDS}

Composite Material, Filemewnt Winding, Pressure Vessels, Pre-stress.

* Phd., Dpt. of Mechatronics, College of Engineering, 6 October University. Please address all correspondence to this author at the above address or by phone at 0127376720 or by E-mail at Essam elmogazy@yahoo.com 


\section{INTRODUCTION}

Composite materials have been used in the industry for over Six decades. One important application of composite materials is in the production of pressure vessels and fluid containers. These structures are characterized by geometries in which the vessels have one (or more) axes of symmetry and the thickness of the composite shell can be considered small in comparison to other dimensions. In general, these composite vessels are produced by a filament winding process. In this process filament bundles saturated with a matrix resin or pre-impregnated filament tapes are wound around a mandrel that defines the finished shape. The filament bundles or tapes must be held in tension during this process to ensure the finished quality. This tension is maintained throughout the winding process and the subsequent cure cycle. As a result, the finished part is preloaded. Many analysis techniques for filament wound systems neglect this preload.

The objective of this paper is to develop a better understanding of the response of filament wound pressure vessels and the effect of preload on the response. The pressure vessels to be considered are thin walled circular cylinders made of laminated composite materials.

\section{THEORITICAL ANALYSIS}

The shell considered in the present work is shown in Fig.1. It is assumed to have an average radius $r$, length $L$, and wall thickness $h$. The coordinate system used in the analysis is also shown in the figure. The displacements of the shell are $u, v$, and $w$ in the $x, \phi$, and $z$ directions, respectively, as shown in the figure.

\section{Strain-Displacement Relationships}

The following assumptions are made based on small deflection theory and the application of the Kirchoff-Love hypothesis:

1. The thickness of the shell, $h$, is very small in comparison with other dimensions, i.e.: $h \ll r$, and $h \ll<$.

2. The normal to the reference middle surface before deformation remains normal after deformation.

3. The normal stress through the thickness is zero.

As a consequence of assumption 2 and for a constant $z$, the in plane shear strains are negligible, i.e.: $\gamma_{x z}=\gamma_{\phi z} \cong 0$. From this result and assumption 3 , the normal strain through the thickness is zero, i.e.: $\varepsilon_{z}=0$. Based on these assumptions, the displacements can be expressed as:

$$
\begin{aligned}
& u=u_{0}-z \frac{\partial w_{0}}{\partial x}, \\
& v=v_{0}-z \frac{\partial w_{0}}{r \partial \phi}, \\
& w=w_{0},
\end{aligned}
$$


where $u_{0}, v_{0}, w_{0}$ are the displacements of the middle surface in the $x, \phi$, and $z$ directions, respectively.

Therefore, from the assumed displacement field and the definition of strain, the straindisplacement relations for any point in the shell are found to be:

$$
\begin{aligned}
\varepsilon_{x} & =\frac{\partial u_{0}}{\partial x}-z \frac{\partial^{2} w_{0}}{\partial x^{2}}, \\
\varepsilon_{\phi} & =\frac{1}{r}\left(\frac{\partial v_{0}}{\partial \phi}-w_{0}\right)-\frac{z}{r^{2}} \frac{\partial^{2} w_{0}}{\partial \phi^{2}}, \\
\gamma_{x \phi} & =\frac{\partial v_{0}}{\partial x}+\frac{1}{r} \frac{\partial u_{0}}{\partial \phi}-\frac{2 z}{r} \frac{\partial^{2} w_{0}}{\partial x \partial \phi} .
\end{aligned}
$$

Which can rewritten in the form:

$$
\begin{aligned}
\varepsilon_{x} & =\varepsilon_{x}^{0}+z \kappa_{x}^{0}, \\
\varepsilon_{\phi} & =\varepsilon_{\phi}^{0}+z \kappa_{\phi}^{0}, \\
\gamma_{x \phi} & =\gamma_{x \phi}^{0}+z \kappa_{x \phi}^{0},
\end{aligned}
$$

where $\varepsilon_{x}^{0}, \varepsilon_{\phi}^{0}, \gamma_{x \phi}^{0}$ are the mid-plane strains and $\kappa_{x}^{0}, \kappa_{\phi}^{0}, \kappa_{x \phi}^{0}$, are the mid-plane curvatures. Note that; $\gamma_{\mathrm{x} \phi}$ is engineering shear strain, not tensorial shear strain.

\section{Stress-Strain Relationships}

In the present work, it is assumed that the shell is in a state of plane stress. Therefore, $\sigma_{z}=\tau_{x z}=\tau_{\phi z}=0$. This assumption is compatible with the previous assumptions. Further, the shell is considered $t$ to be made of orthotropic lamina. Therefore, each lamina of the shell has a set of principal material directions. These directions form a local coordinate system in the lamina, i.e.: 1-, 2-, z directions. Under the plane stress state, the stress-strain relationships for the kth lamina in the local lamina coordinate directions are:

$$
\left\{\begin{array}{l}
\sigma_{1} \\
\sigma_{2} \\
\tau_{12}
\end{array}\right\}_{k}=\left[\begin{array}{ccc}
Q_{11} & Q_{12} & 0 \\
Q_{21} & Q_{22} & 0 \\
0 & 0 & Q_{66}
\end{array}\right]_{k}\left\{\begin{array}{c}
\varepsilon_{1} \\
\varepsilon_{2} \\
\gamma_{12}
\end{array}\right\}_{k},
$$

where $Q_{i j}$ are the principal stiffnesses of the kth lamina, $\sigma_{i}$ and $\tau_{12}$ are the normal and shear stresses in the principal material directions, respectively, and $\varepsilon_{i}$ and $\gamma_{12}$ the normal and shear strains in the principal material directions, respectively. The notation used in this paper is taken from that used by Jones [1]. These stiffness terms are 
directly related to the engineering properties (moduli and Poisson's ratio) of the lamina. Expressions for these terms can be found in various text books [1, 2, and 3].

Through a coordinate transformation, the stress-strain relationships for the kth lamina in the global coordinate system of the shell can be expressed as:

$$
\left\{\begin{array}{c}
\sigma_{x} \\
\sigma_{\phi} \\
\tau_{x \phi}
\end{array}\right\}_{k}=\left[\begin{array}{lll}
\bar{Q}_{11} & \bar{Q}_{12} & \bar{Q}_{16} \\
\bar{Q}_{21} & \bar{Q}_{22} & \bar{Q}_{26} \\
\bar{Q}_{61} & \bar{Q}_{62} & \bar{Q}_{66}
\end{array}\right]_{k}\left\{\begin{array}{c}
\varepsilon_{x} \\
\varepsilon_{\phi} \\
\gamma_{x \phi}
\end{array}\right\}_{k},
$$

where $\bar{Q}_{i j}$ are the stiffnesses of the $k$ th lamina in the global coordinate system. Expressions for these terms in terms of $\theta_{k}$, the angle between the first principal material direction and the $x$ direction, the winding angle, can be found in various text books [1,2, and 3]. The stress in the $k$ th lamina may then be expressed in terms of the mid-plane strains and curvatures by substituting equation (2) into equation (5). Thus producing the lamina stress-strain/curvature relationships:

$$
\left\{\begin{array}{c}
\sigma_{x} \\
\sigma_{\phi} \\
\tau_{x \phi}
\end{array}\right\}_{k}=[\bar{Q}]_{k}\left\{\begin{array}{c}
\varepsilon_{x}^{0} \\
\varepsilon_{\phi}^{0} \\
\gamma_{x \phi}^{0}
\end{array}\right\}+z[\bar{Q}]_{k}\left\{\begin{array}{c}
\kappa_{x}^{0} \\
\kappa_{\phi}^{0} \\
\kappa_{x \phi}^{0}
\end{array}\right\} .
$$

\section{Force and Moment Resultants}

Consider the laminated cylindrical shell to have $n$ laminae. Further, let a typical lamina, $k$, be bounded by the surfaces $z=h_{k-1}$ and $z=h_{k}$. As a consequence of the assumption that $\frac{h}{r}<<1$, the force and moment resultants can be written as:

$$
\begin{aligned}
& \left\{\begin{array}{c}
N_{x}^{o} \\
N_{\phi}^{o} \\
N_{x \phi}^{o}
\end{array}\right\}=\sum_{k=1}^{n} \int_{h_{k-1}}^{h_{k}}\left\{\begin{array}{l}
\sigma_{x} \\
\sigma_{\phi} \\
\tau_{x \phi}
\end{array}\right\}_{k} d z, \\
& \left\{\begin{array}{l}
M_{x}^{o} \\
M_{\phi}^{o} \\
M_{x \phi}^{o}
\end{array}\right\}=\sum_{k=1}^{n} \int_{h_{k-1}}^{h_{k}}\left\{\begin{array}{l}
\sigma_{x} \\
\sigma_{\phi} \\
\tau_{x \phi}
\end{array}\right\}_{k} z d z,
\end{aligned}
$$

where $N_{x}^{o}$ and $N_{\phi}^{o}$ are defined as the normal in-plane forces per unit length in the $x$, and $\phi$ directions respectively, $N_{x \phi}^{o}$ is defined as the in-plane shear force per unit length 
in the $x$ - $\phi$ plane, $M_{x}^{o}$ and $M_{\phi}^{o}$ are defined as the bending moments per unit length in the $x$, and $\phi$ directions respectively, and $M_{x \phi}^{o}$ is defined as the twisting moment per unit length in the $x$ - $\phi$ plane.

Finally, the relationships between the force and moment resultants and the strains and curvatures of the mid-plane are obtained by substituting the results found in equation (6) into equations (7) and (8). Noting that the mid-plane strains and curvatures, $\left\{\varepsilon^{0}\right\}$ and $\left\{\kappa^{0}\right\}$ are independent of the variable of integration and the stiffnesses, $[\bar{Q}]_{k}$, are constant for each lamina, this relation becomes:

$$
\left\{\begin{array}{l}
N^{o} \\
\hline M^{o}
\end{array}\right\}=\left[\begin{array}{l|l}
A & B \\
\hline B & D
\end{array}\right]\left\{\begin{array}{l}
\frac{\varepsilon^{0}}{\kappa^{0}}
\end{array}\right\}
$$

where:

$$
\begin{aligned}
A_{i j} & =\sum_{k=1}^{n} \bar{Q}_{i j}\left(h_{k-1}-h_{k}\right), i, j=1,2,6, \\
B_{i j} & =\frac{1}{2} \sum_{k=1}^{n} \bar{Q}_{i j}\left(h_{k-1}^{2}-h_{k}^{2}\right), \\
D_{i j} & =\frac{1}{3} \sum_{k=1}^{n} \bar{Q}_{i j}\left(h_{k-1}^{3}-h_{k}^{3}\right),
\end{aligned}
$$

which is as found in classical lamination theory.

\section{PRESTRESS AND THE FORCE AND MOMENT RESULTANTS}

During the winding process, the filaments that make up the lamina are wrapped or wound around a mandrel. This process requires a tension be maintained on the filament bundle or tow throughout the winding process. The result is a preload or prestress in each lamina of the shell. The prestress or residual stress remaining in the part after all processing is dependent on a number of factors which include: shell geometry, fiber tension, wrap angle, relative speed between the mandrel and feeder, resin viscosity, cure method, and cure process or cycle. For the purposes of this analysis it is assumed that the post-processing tension in a fiber bundle or tow is constant along the length of the bundle within a given lamina. This tension is denoted by $T$. Therefore, the resulting prestress in the lamina in the local coordinate system is: 


$$
\left\{\begin{array}{c}
\sigma_{1}^{*} \\
\sigma_{2}^{*} \\
\tau_{12}^{*}
\end{array}\right\}=\left\{\begin{array}{c}
\sigma_{T} \\
0 \\
0
\end{array}\right\}=\left\{\begin{array}{c}
T / A_{f} \\
0 \\
0
\end{array}\right\},
$$

where $\sigma_{1}^{*}, \sigma_{2}^{*}$, and $\tau_{12}^{*}$ are the components of the prestress in the principal material directions, $\sigma_{T}$ is the stress on the fiber bundle, and $A_{f}$ is the cross-sectional area of the fiber bundle.

Given the wrap angle, defined as $\theta_{k}$, is constant for the $k$ th lamina, the prestress can be transformed into the global coordinate system. The result of this transform is:

$$
\left\{\begin{array}{c}
\sigma_{x}^{*} \\
\sigma_{y}^{*} \\
\tau_{x y}^{*}
\end{array}\right\}=\sigma_{T}\left\{\begin{array}{c}
\cos ^{2} \theta_{k} \\
\sin ^{2} \theta_{k} \\
\sin \theta_{k} \cos \theta_{k}
\end{array}\right\},
$$

where $\sigma_{T}$ is the prestress in the $k$ th lamina. The force and bending moment resultants due to the prestress can be found by substituting equation (12) into equations (7) and (8). The result is:

$$
\begin{aligned}
& \left\{\begin{array}{c}
N_{x}^{*} \\
N_{y}^{*} \\
N_{x \phi}^{*}
\end{array}\right\}=\int_{-h / 2}^{h / 2}\left\{\begin{array}{c}
\sigma_{x}^{*} \\
\sigma_{\phi}^{*} \\
\tau_{x \phi}^{*}
\end{array}\right\} d z=\sum_{k=1}^{n} \sigma_{T}\left\{\begin{array}{c}
\cos ^{2} \theta \\
\sin ^{2} \theta \\
\cos \theta \sin \theta
\end{array}\right\}_{k}\left(h_{k-1}-h_{k}\right) \\
& \left\{\begin{array}{l}
M_{x}^{*} \\
M_{\phi}^{*} \\
M_{x \phi}^{*}
\end{array}\right\}=\int_{-h / 2}^{h / 2}\left\{\begin{array}{c}
\sigma_{x}^{*} \\
\sigma_{\phi}^{*} \\
\tau_{x \phi}^{*}
\end{array}\right\} z d z=\frac{1}{2} \sum_{k=1}^{n} \sigma_{T}\left\{\begin{array}{c}
\cos ^{2} \theta \\
\sin ^{2} \theta \\
\cos \theta \sin \theta
\end{array}\right\}_{k}\left(h_{k-1}^{2}-h_{k}^{2}\right)
\end{aligned}
$$

where ${ }^{*}$ designate the resultants due to the prestress terms. These prestress terms exist in the shell in the undeformed or reference configuration. Therefore, the laminate constitutive relation as given in equation (9) now takes the form:

$$
\left\{\begin{array}{l}
N \\
\hline M
\end{array}\right\}=\left[\begin{array}{l|l}
A & B \\
\hline B & D
\end{array}\right]\left\{\frac{\varepsilon^{0}}{\kappa^{0}}\right\}+\left\{\frac{N^{*}}{M^{*}}\right\} .
$$




\section{EQUILIBRIUM EQUATIONS FOR LAMINATED CYLINDERICAL SHELL}

Consider an infinitesimal element from a laminated cylindrical shell. This element is cut from the shell by two adjacent axial sections and two adjacent sections perpendicular to the axis of the cylinder, as shown in Fig.1. The corresponding element of the middle surface of the shell is shown in Fig.2 in its deformed configuration. In Fig.3, the force and bending moment resultants are designated as $\bar{N}_{i}, \bar{N}_{i j}, \bar{M}_{i}$, and $\bar{M}_{i j}$, differentiating them from the resultants in the undeformed configuration.

From Timeoshenko and Woinowsky-Krieger [4], Timoshenko and Gere [5], and the assumption that $\frac{h}{r}<<1$, then $\bar{N}_{x \phi} \neq \bar{N}_{\phi x}, \bar{M}_{x \phi} \neq \bar{M}_{\phi x}$, and the force equilibrium equations are:

$$
\begin{aligned}
& \frac{\partial \bar{N}_{x}}{\partial x}+\frac{1}{r} \frac{\partial \bar{N}_{\phi x}}{\partial \phi}-\bar{N}_{x z} \frac{\partial^{2} w_{0}}{\partial x^{2}}-\bar{N}_{x \phi} \frac{\partial^{2} v_{0}}{\partial x^{2}}-\frac{\bar{N}_{\phi z}}{r}\left(\frac{\partial v_{0}}{\partial x}+\frac{\partial^{2} w_{0}}{\partial x \partial \phi}\right) \bar{N}_{\phi}\left(\frac{\partial^{2} v_{0}}{\partial x \partial \phi}-\frac{\partial w_{0}}{\partial x}\right)+\bar{p}_{x}=0, \\
& \frac{1}{r} \frac{\partial \bar{N}_{\phi}}{\partial \phi}+\frac{\partial \bar{N}_{x \phi}}{\partial x}+\bar{N}_{x} \frac{\partial^{2} v_{0}}{\partial x^{2}}-\frac{\bar{N}_{x z}}{r}\left(\frac{\partial v_{0}}{\partial x}+\frac{\partial^{2} w_{0}}{\partial x \partial \phi}\right)+\frac{\bar{N}_{\phi x}}{r}\left(\frac{\partial^{2} v_{0}}{\partial x \partial \phi}-\frac{\partial w_{0}}{\partial x}\right) \\
&-\frac{\bar{N}_{\phi z}}{r}\left(1+\frac{1}{r} \frac{\partial v_{0}}{\partial \phi}+\frac{1}{r} \frac{\partial^{2} w_{0}}{\partial \phi^{2}}\right)+\bar{p}_{\phi}=0, \\
& \frac{\partial \bar{N}_{x z}}{\partial x}+\frac{1}{r} \frac{\partial \bar{N}_{\phi z}}{\partial \phi}+\frac{\left(\bar{N}_{x \phi}+\bar{N}_{\phi x}\right)}{r}\left(\frac{\partial v_{0}}{\partial x}+\frac{\partial^{2} w_{0}}{\partial x \partial \phi}\right)+\bar{N}_{x} \frac{\partial^{2} w_{0}}{\partial x^{2}} \\
&+\frac{\bar{N}_{\phi}}{r}\left(1+\frac{1}{r} \frac{\partial v_{0}}{\partial \phi}+\frac{1}{r} \frac{\partial^{2} w_{0}}{\partial \phi^{2}}\right)+\bar{p}_{z}=0,
\end{aligned}
$$

Further, the moment equilibrium equations are:

$$
\begin{aligned}
& \frac{\partial \bar{M}_{x \phi}}{\partial x}-\frac{1}{r} \frac{\partial \bar{M}_{\phi}}{\partial \phi}-\frac{\bar{M}_{\phi x}}{r}\left(\frac{\partial^{2} v_{0}}{\partial x \partial \phi}-\frac{\partial w_{0}}{\partial x}\right)-\bar{M}_{x} \frac{\partial^{2} v_{0}}{\partial x^{2}}+\bar{N}_{\phi z}=0, \\
& \frac{1}{r} \frac{\partial \bar{M}_{\phi x}}{\partial \phi}+\frac{\partial \bar{M}_{x}}{\partial x}+\bar{M}_{x \phi} \frac{\partial^{2} v_{0}}{\partial x^{2}}-\frac{\bar{M}_{\phi}}{r}\left(\frac{\partial^{2} v_{0}}{\partial x \partial \phi}-\frac{\partial w_{0}}{\partial x}\right)-\bar{N}_{x z}=0,
\end{aligned}
$$




$$
\begin{aligned}
\frac{\bar{M}_{x}}{r}\left(\frac{\partial v_{0}}{\partial x}+\frac{\partial^{2} w_{0}}{\partial x \partial \phi}\right)+\bar{M}_{\phi x}\left(\frac{1}{r}+\frac{1}{r^{2}} \frac{\partial v_{0}}{\partial \phi}+\frac{1}{r^{2}} \frac{\partial^{2} w_{0}}{\partial \phi^{2}}\right)+\bar{M}_{x \phi} \frac{\partial^{2} w_{0}}{\partial x^{2}}-\frac{\bar{M}_{\phi}}{r}\left(\frac{\partial v_{0}}{\partial x}+\frac{\partial^{2} w_{0}}{\partial x \partial \phi}\right) \\
+\bar{N}_{x \phi}-\bar{N}_{\phi x}=0 .
\end{aligned}
$$

\section{Static Response of Laminated Cylinderical Shells Under Uniform Pressure}

The set of differential equations (16) through (21) represent the equilibrium of cylindrical shells in terms of the internal resultant forces and moments in the deformed configuration, and the displacements of the middle surface. To obtain the displacement field; equations (16) through (21) must be solved simultaneously. Alternatively, the force / moment -- displacement / curvature relations may be substituted into equations (16) through (21) to obtain a set of simultaneous partial differential equations in the displacements of the mid-plane.

Consider at this point the case of static response to uniform lateral pressure (internal or external pressure on the shell in the radial, i.e.: z-direction). The lateral compressive pressure is $p_{z}=p$. This load is constant, therefore, the resultant shear forces are very small compared to the other forces. Consequently, the products of these forces with displacement terms and derivatives of displacement are negligible. Hence, the force balance equations (16) through (18), take the following form:

$$
\begin{aligned}
& \frac{\partial \bar{N}_{x}}{\partial x}+\frac{1}{r} \frac{\partial \bar{N}_{\phi x}}{\partial \phi}-\bar{N}_{x \phi} \frac{\partial^{2} v_{0}}{\partial x^{2}}-\frac{\bar{N}_{\phi}}{r}\left(\frac{\partial^{2} v_{0}}{\partial x \partial \phi}-\frac{\partial w_{0}}{\partial x}\right)=0 \\
& \frac{1}{r} \frac{\partial \bar{N}_{\phi}}{\partial \phi}+\frac{\partial \bar{N}_{x \phi}}{\partial x}+\bar{N}_{x} \frac{\partial^{2} v_{0}}{\partial x^{2}}+\frac{\bar{N}_{\phi x}}{r}\left[\frac{\partial^{2} v_{0}}{\partial x \partial \phi}-\frac{\partial w_{0}}{\partial x}\right]-\frac{\bar{N}_{\phi z}}{r}=0 \\
& \frac{\partial \bar{N}_{x z}}{\partial x}+\frac{1}{r} \frac{\partial \bar{N}_{\phi z}}{\partial \phi}+\frac{\bar{N}_{x \phi}+\bar{N}_{\phi x}}{r}\left(\frac{\partial v_{0}}{\partial x}+\frac{\partial^{2} w_{0}}{\partial x \partial \phi}\right)+\bar{N}_{x} \frac{\partial^{2} w_{0}}{\partial x^{2}}+\frac{\bar{N}_{\phi}}{r}\left(1+\frac{1}{r} \frac{\partial v_{0}}{\partial \phi}+\frac{1}{r} \frac{\partial^{2} w_{0}}{\partial \phi^{2}}\right)+\bar{p}=0 .
\end{aligned}
$$

By substituting the moment equilibrium equations, equations (19) and (20) into the $\phi$ and $z$ - direction force equilibrium equations, equations (23) and (24) the governing equations for this case reduce to:

$$
\begin{aligned}
\frac{\partial \bar{N}_{x}}{\partial x}+ & \frac{1}{r} \frac{\partial \bar{N}_{\phi x}}{\partial \phi}-\bar{N}_{x \phi} \frac{\partial^{2} v_{0}}{\partial x^{2}}-\frac{\bar{N}_{\phi}}{r}\left(\frac{\partial^{2} v_{0}}{\partial x \partial \phi}-\frac{\partial w_{0}}{\partial x}\right)=0, \\
\frac{1}{r} \frac{\partial \bar{N}_{\phi}}{\partial \phi}+ & \frac{\partial \bar{N}_{x \phi}}{\partial x}+\bar{N}_{x} \frac{\partial^{2} v_{0}}{\partial x^{2}}+\frac{\bar{N}_{\phi x}}{r}\left(\frac{\partial^{2} v_{0}}{\partial x \partial \phi}-\frac{\partial w_{0}}{\partial x}\right) \\
& +\frac{1}{r}\left(\partial \frac{\partial \bar{M}_{x \phi}}{\partial x}-\frac{1}{r} \partial \frac{\partial \bar{M}_{\phi}}{\partial \phi}-\frac{\bar{M}_{\phi x}}{r}\left(\frac{\partial^{2} v_{0}}{\partial x \partial \phi}-\frac{\partial w_{0}}{\partial x}\right)-\bar{M}_{x} \frac{\partial^{2} v_{0}}{\partial x^{2}}\right)=0,
\end{aligned}
$$




$$
\begin{aligned}
\frac{\partial^{2} \bar{M}_{x}}{\partial x^{2}}+ & \frac{1}{r}\left(\frac{\partial^{2} \bar{M}_{\phi x}}{\partial x \partial \phi}-\frac{\partial^{2} \bar{M}_{x \phi}}{\partial x \partial \phi}\right)+\frac{1}{r^{2}} \frac{\partial^{2} \bar{M}_{\phi}}{\partial \phi^{2}}+\left(\frac{\partial \bar{M}_{x \phi}}{\partial x}+\frac{1}{r} \frac{\partial \bar{M}_{x}}{\partial \phi}\right) \frac{\partial^{2} v_{0}}{\partial x^{2}} \\
& -\frac{1}{r}\left(\frac{\partial \bar{M}_{\phi}}{\partial x}-\frac{1}{r} \frac{\partial \bar{M}_{\phi x}}{\partial \phi}\right)\left(\frac{\partial^{2} v_{0}}{\partial x \partial \phi}-\frac{\partial w_{0}}{\partial x}\right)+\frac{\bar{M}_{x}}{r} \frac{\partial^{3} v_{0}}{\partial x^{2} \partial \phi}+\bar{M}_{x \phi} \frac{\partial^{3} v_{0}}{\partial x^{3}} \\
& +\frac{\bar{M}_{\phi x}}{r^{2}}\left(\frac{\partial^{3} v_{0}}{\partial x \partial \phi^{2}}-\frac{\partial^{2} w_{0}}{\partial x \partial \phi}\right)-\frac{\bar{M}_{\phi}}{r}\left(\frac{\partial^{3} v_{0}}{\partial x^{2} \partial \phi}-\frac{\partial^{2} w_{0}}{\partial x^{2}}\right) \\
& +\frac{\bar{N}_{\phi x}+\bar{N}_{x \phi}}{r}\left(\frac{\partial v_{0}}{\partial x}+\frac{\partial^{2} w_{0}}{\partial x \partial \phi}\right)+\bar{N}_{x} \frac{\partial^{2} w_{0}}{\partial x^{2}}+\frac{\bar{N}_{\phi}}{r}\left(1+\frac{1}{r} \frac{\partial v_{0}}{\partial \phi}+\frac{1}{r} \frac{\partial^{2} w_{0}}{\partial \phi^{2}}\right)+\bar{p}=0 .
\end{aligned}
$$

$$
\begin{aligned}
\frac{\bar{M}_{x}}{r}\left(\frac{\partial v_{0}}{\partial x}+\frac{\partial^{2} w_{0}}{\partial x \partial \phi}\right)+\bar{M}_{\phi x}\left(\frac{1}{r}+\frac{1}{r^{2}} \frac{\partial v_{0}}{\partial \phi}+\frac{1}{r^{2}} \frac{\partial^{2} w_{0}}{\partial \phi^{2}}\right)+\bar{M}_{x \phi} \frac{\partial^{2} w_{0}}{\partial x^{2}}-\frac{\bar{M}_{\phi}}{r}\left(\frac{\partial v_{0}}{\partial x}+\frac{\partial^{2} w_{0}}{\partial x \partial \phi}\right) \\
+\bar{N}_{x \phi}-\bar{N}_{\phi x}=0 .
\end{aligned}
$$

Equations (25) through (28) are the complete equilibrium equations for the static response of a laminated cylindrical shell under uniform lateral pressure.

Applying Timoshenko approach [5], the stretching of the middle surface of the shell can be taken into account by defining the following relations:

$$
\begin{aligned}
\bar{N}_{x} & =N_{x}\left(1+\varepsilon_{\phi}^{0}\right), \\
\bar{N}_{\phi} & =\left(N_{\phi}-p r\right)\left(1+\varepsilon_{x}^{0}\right), \\
\bar{N}_{x \phi} & =N_{x \phi}\left(1+\varepsilon_{\phi}^{0}\right), \\
\bar{N}_{\phi x} & =N_{x \phi}\left(1+\varepsilon_{x}^{0}\right), \\
\bar{M}_{x} & =M_{x}\left(1+\varepsilon_{\phi}^{0}\right), \\
\bar{M}_{\phi} & =M_{\phi}\left(1+\varepsilon_{x}^{0}\right), \\
\bar{M}_{x \phi} & =M_{x \phi}\left(1+\varepsilon_{\phi}^{0}\right), \\
\bar{M}_{\phi x} & =M_{x \phi}\left(1+\varepsilon_{x}^{0}\right), \\
\bar{p} & =p\left(1+\varepsilon_{x}^{0}\right)\left(1+\varepsilon_{\phi}^{0}\right),
\end{aligned}
$$

where: 


$$
\begin{aligned}
& N_{x}=N_{x}^{o}+N_{x}^{*}, \\
& N_{\phi}=N_{\phi}^{o}+N_{\phi}^{*}, \\
& N_{x \phi}=N_{x \phi}^{o}+N_{x \phi}^{*}, \\
& M_{x}=M_{x}^{o}+M_{x}^{*}, \\
& M_{\phi}=M_{\phi}^{o}+M_{\phi}^{*}, \\
& M_{x \phi}=M_{x \phi}^{o}+M_{x \phi}^{*} .
\end{aligned}
$$

Recall that $N_{i}^{o}$ and $M_{i}^{o}$ are the resultant forces and moments in the undeformed geometry, respectively, and $N_{i}^{*}$ and $M_{i}^{*}$ are the prestress resultant forces and moments in the undeformed geometry, respectively. The governing equations for equilibrium of the shell in terms of the variables in the undeformed configuration are found by substituting the definitions from equation (29) into equations (25) through (28). After rearranging these equations and noting the assumptions on laminate configuration are such that the resultant forces and moments due to the prestress are independent of $x$ and $\phi$, the following equations are obtained:

$$
\begin{aligned}
& \frac{\partial N_{x}^{o}}{\partial x}(1+\left.\frac{1}{r} \frac{\partial v_{0}}{\partial \phi}-\frac{w_{0}}{r}\right)+\frac{1}{r} \frac{\partial N_{x \phi}^{o}}{\partial \phi}\left(1+\frac{\partial u_{0}}{\partial x}\right) \\
&+\left(p+\frac{\left(N_{x}^{o}-N_{\phi}^{o}\right)+\left(N_{x}^{*}-N_{\phi}^{*}\right)}{r}+\frac{\partial u_{0}}{\partial x}\left(p-\frac{N_{\phi}^{o}+N_{\phi}^{*}}{r}\right)\right)\left(\frac{\partial^{2} v_{0}}{\partial x \partial \phi}-\frac{\partial w_{0}}{\partial x}\right) \\
&+\left(\frac{N_{x \phi}^{o}+N_{x \phi}^{*}}{r}\right)\left(\frac{\partial^{2} u_{0}}{\partial x \partial \phi}-\left(r+\frac{\partial v_{0}}{\partial \phi}-w_{0}\right) \frac{\partial^{2} v_{0}}{\partial x^{2}}\right)=0 \\
& \frac{1}{r}\left(\frac{\partial \partial N_{\phi}^{o}}{\partial \phi}-\frac{1}{r} \frac{\partial M_{\phi}^{o}}{\partial \phi}\right)\left(1+\frac{\partial u_{0}}{\partial x}\right)+\left(\frac{\partial N_{x \phi}^{o}}{\partial x}-\frac{1}{r} \frac{\partial M_{x \phi}^{o}}{\partial x}\right)\left(1+\frac{1}{r} \frac{\partial v_{0}}{\partial \phi}-\frac{w_{0}}{r}\right) \\
&+\left(\frac{N_{\phi}^{o}+N_{\phi}^{*}}{r}-\frac{M_{\phi}^{o}+M_{\phi}^{*}}{r^{2}}-p\right) \frac{\partial^{2} u_{0}}{\partial x \partial \phi} \\
&+\frac{\left(N_{x \phi}^{o}+N_{x \phi}^{*}\right)}{r}\left(\frac{\partial^{2} v_{0}}{\partial x \partial \phi}-\frac{\partial w_{0}}{\partial x}\right)\left(2+\frac{\partial u_{0}}{\partial x}\right) \\
&+\left(N_{x}^{o}+N_{x}^{*}-\frac{M_{x}^{o}+M_{x}^{*}}{r}\right)\left(\frac{\partial^{2} v_{0}}{\partial x^{2}}\right)\left(1+\frac{1}{r} \frac{\partial v_{0}}{\partial \phi}-\frac{w_{0}}{r}\right) \\
&-\frac{M_{\phi}^{o}+M_{\phi}^{*}}{r^{2}}\left(\frac{\partial u_{0}}{\partial x}\right)\left(\frac{\partial^{2} v_{0}}{\partial x \partial \phi}-\frac{\partial w_{0}}{\partial x}\right)=0,
\end{aligned}
$$




$$
\begin{aligned}
& \left(\frac{M_{x}^{o}+M_{x}^{*}}{r}\left(1+\frac{1}{r} \frac{\partial v_{0}}{\partial \phi}-\frac{w_{0}}{r}\right)-\frac{M_{\phi}^{o}+M_{\phi}^{*}}{r}\left(1+\frac{\partial u_{0}}{\partial x}\right)\right)\left(\frac{\partial v_{0}}{\partial x}+\frac{\partial^{2} w_{0}}{\partial x \partial \phi}\right) \\
& +\frac{M_{x \phi}^{o}+M_{x \phi}^{*}}{r}\left(\begin{array}{c}
\left(1+\frac{\partial u_{0}}{\partial x}\right)\left(1+\frac{1}{r} \frac{\partial v_{0}}{\partial x}-\frac{1}{r} \frac{\partial^{2} w_{0}}{\partial \phi^{2}}\right) \\
+\frac{\partial^{2} w_{0}}{\partial x^{2}}\left(1+\frac{1}{r} \frac{\partial v_{0}}{\partial \phi}-\frac{w_{0}}{r}\right)
\end{array}\right) \\
& +\left(N_{x \phi}^{o}+N_{x \phi}^{*}\right)\left(\frac{\partial u_{0}}{\partial x}+\frac{1}{r} \frac{\partial v_{0}}{\partial \phi}-\frac{w_{0}}{r}\right)=0
\end{aligned}
$$




$$
\begin{aligned}
& \frac{\partial^{2} M_{x}^{o}}{\partial x^{2}}\left(1+\frac{1}{r} \frac{\partial v_{0}}{\partial \phi}-\frac{w_{0}}{r}\right)+\frac{1}{r^{2}} \frac{\partial^{2} M_{\phi}^{o}}{\partial \phi^{2}}\left(1+\frac{\partial u_{0}}{\partial x}\right)+\frac{1}{r} \frac{\partial^{2} M_{x \phi}^{o}}{\partial x \partial \phi}\left(\frac{\partial u_{0}}{\partial x}+\frac{w_{0}}{r}-\frac{1}{r} \frac{\partial v_{0}}{\partial \phi}\right) \\
& +\frac{2}{r} \frac{\partial M_{x}^{o}}{\partial x}\left(\frac{\partial^{2} v_{0}}{\partial x \partial \phi}-\frac{\partial w_{0}}{\partial x}\right)+\frac{1}{r} \frac{\partial M_{x}^{o}}{\partial \phi}\left(\frac{\partial^{2} v_{0}}{\partial x^{2}}\right)\left(1+\frac{1}{r} \frac{\partial v_{0}}{\partial \phi}-\frac{w_{0}}{r}\right)+\frac{2}{r^{2}} \frac{\partial M_{\phi}^{o}}{\partial \phi} \\
& +\frac{1}{r} \frac{\partial M_{\phi}^{o}}{\partial x}\left(\left(\frac{\partial w_{0}}{\partial x}-\frac{\partial^{2} v_{0}}{\partial x \partial \phi}\right)\left(1+\frac{\partial u_{0}}{\partial x}\right)\right) \\
& +\frac{\partial M_{x \phi}^{o}}{\partial x}\left(\frac{1}{r}\left(\frac{\partial^{2} u_{0}}{\partial x \partial \phi}-\frac{1}{r} \frac{\partial^{2} v_{0}}{\partial \phi^{2}}+\frac{1}{r} \frac{\partial w_{0}}{\partial \phi}\right)+\frac{\partial^{2} v_{0}}{\partial x^{2}}\left(1+\frac{w_{0}}{r}-\frac{1}{r} \frac{\partial v_{0}}{\partial \phi}\right)\right) \\
& +\frac{\partial M_{x \phi}^{o}}{\partial \phi}\left(\frac{1}{r}\left(\frac{\partial^{2} u_{0}}{\partial x^{2}}-\frac{1}{r} \frac{\partial w_{0}}{\partial x}-\frac{1}{r} \frac{\partial^{2} v_{0}}{\partial x \partial \phi}\right)+\frac{1}{r^{2}}\left(\frac{\partial^{2} v_{0}}{\partial x \partial \phi}-\frac{\partial w_{0}}{\partial x}\right)\left(1+\frac{\partial u_{0}}{\partial x}\right)\right) \\
& +\frac{M_{x}^{o}+M_{x}^{*}}{r}\left(\left(2+\frac{1}{r} \frac{\partial v_{0}}{\partial \phi}-\frac{w_{0}}{r}\right) \frac{\partial^{3} v_{0}}{\partial x^{2} \partial \phi}+\frac{1}{r}\left(\frac{\partial^{2} v_{0}}{\partial \phi^{2}}-\frac{\partial w_{0}}{\partial \phi}\right) \frac{\partial^{2} v_{0}}{\partial x^{2}}-\frac{\partial^{2} w_{0}}{\partial x^{2}}\right) \\
& +\frac{M_{\phi}^{o}+M_{\phi}^{*}}{r}\left(\frac{1}{r} \frac{\partial^{3} u_{0}}{\partial x \partial \phi^{2}}+\frac{\partial^{2} u_{0}}{\partial x^{2}}\left(\frac{\partial w_{0}}{\partial x}-\frac{\partial^{2} v_{0}}{\partial x \partial \phi}\right)+\left(1+\frac{\partial u_{0}}{\partial x}\right)\left(\frac{\partial^{2} w_{0}}{\partial x^{2}}-\frac{\partial^{3} v_{0}}{\partial x^{2} \partial \phi}\right)\right) \\
& +\frac{M_{x \phi}^{o}+M_{x \phi}^{*}}{r}\left(\begin{array}{l}
\frac{\partial^{3} u_{0}}{\partial x^{2} \partial \phi}+\frac{1}{r}\left(\frac{\partial^{3} v_{0}}{\partial x \partial \phi^{2}}-\frac{\partial^{2} w_{0}}{\partial x \partial \phi}\right) \frac{\partial u_{0}}{\partial x} \\
\left.+\left(\frac{1}{r} \frac{\partial^{2} u_{0}}{\partial x \partial \phi}+\frac{\partial^{2} v_{0}}{\partial x^{2}}\right)\left(\frac{\partial^{2} v_{0}}{\partial x \partial \phi}-\frac{\partial w_{0}}{\partial x}\right)+\left(r+\frac{\partial v_{0}}{\partial \phi}-w_{0}\right) \frac{\partial^{3} v_{0}}{\partial x^{3}}\right)
\end{array}\right. \\
& +\left(N_{x}^{o}+N_{x}^{*}\right)\left(1+\frac{1}{r} \frac{\partial v_{0}}{\partial \phi}-\frac{w_{0}}{r}\right) \frac{\partial^{2} w_{0}}{\partial x^{2}}+\frac{N_{\phi}^{o}+N_{\phi}^{*}}{r}\left(1+\frac{1}{r} \frac{\partial v_{0}}{\partial \phi}+\frac{1}{r} \frac{\partial^{2} w_{0}}{\partial \phi^{2}}\right) \\
& +\frac{N_{x \phi}^{o}+N_{x \phi}^{*}}{r}\left(2+\frac{\partial u_{0}}{\partial x}+\frac{1}{r} \frac{\partial v_{0}}{\partial \phi}-\frac{w_{0}}{r}\right)\left(\frac{\partial v_{0}}{\partial x}+\frac{\partial^{2} w_{0}}{\partial x \partial \phi}\right) \\
& +p\left(\frac{\partial u_{0}}{\partial x}\left(1-\frac{1}{r} \frac{\partial v_{0}}{\partial \phi}-\frac{w_{0}}{r}\right)-\frac{1}{r}\left(w_{0}+\frac{\partial^{2} w_{0}}{\partial \phi^{2}}\right)\right)=0
\end{aligned}
$$

To further reduce these equations to a system of partial differential equations to expressions involving only the displacements of the middle surface, it is necessary to substitute the results from equations (2) and (9). This results in a set of simultaneous partial differential equations for the mid-surface deflections for a laminated cylindrical shell under constant uniform lateral pressure. These equations are further simplified by 
assuming that the deformation is axisymmetric. This assumption is based on the facts that:

(1) the load and boundary conditions are independent of $\phi$,

(2) the material used is uniform and homogeneous in the $\phi$-direction, and

(3) the material stiffnesses, $A_{i j}, B_{i j}$, and $D_{i j}$, are independent of $x, \phi$, and $z$.

Consequently, the circumferential displacement, $v_{0}$, is zero or constant, and that $u_{0}$ and $w_{0}$ are functions of $x$ alone (i.e.: independent of $\phi$ ). Thus, all partial derivatives of $u_{0}$ and $w_{0}$ with respect to $\phi$ vanish. Then equations (30) through (33) using equations (2) and (9) reduce to a set of four simultaneous nonlinear ordinary differential equations in the mid-surface displacements, $u_{0}$ and $w_{0}$. These equations are:

$$
\begin{aligned}
& A_{11} u_{0}^{\prime \prime}-B_{11} w_{0}^{\prime \prime \prime}-\left[\frac{\left(A_{12}+N_{x}^{*}-N_{\phi}^{*}\right)}{r}+p\right] w_{0}^{\prime}+\left(\frac{N_{\phi}^{*}+A_{12}-A_{11}}{r}-p\right) u_{0}^{\prime} w_{0}^{\prime} \\
& -\frac{A_{11}}{r} u_{0}^{\prime \prime} w_{0}+\frac{B_{11}-B_{12}}{r} w_{0}^{\prime} w_{0}^{\prime \prime}+\frac{B_{11}}{r} w_{0} w_{0}^{\prime \prime \prime}+\frac{2 A_{12}-A_{22}}{r^{2}} w_{0} w_{0}^{\prime} \\
& +\frac{A_{12}}{r} u_{0}^{\prime 2} w_{0}^{\prime}-\frac{A_{22}}{r^{2}} u_{0}^{\prime} w_{0} w_{0}^{\prime}-\frac{B_{12}}{r} u_{0}^{\prime} w_{0}^{\prime} w_{0}^{\prime \prime}=0, \\
& \left(A_{16}+\frac{B_{16}}{r}\right) u_{0}^{\prime \prime}-\frac{1}{r}\left[A_{26}+\frac{B_{26}}{r}-2 N_{x \phi}^{*}\right] w_{0}^{\prime}-\left(B_{16}+\frac{D_{16}}{r}\right) w_{0}^{\prime \prime \prime} \\
& +\frac{1}{r}\left(\frac{M_{x \phi}^{*}}{r}-N_{x \phi}^{*}-2 A_{16}\right) u_{0}^{\prime} w_{0}^{\prime}-\frac{1}{r}\left(A_{16}+\frac{B_{16}}{r}\right) u_{0}^{\prime \prime} w_{0} \\
& -\frac{1}{r^{2}}\left(3 A_{26}+\frac{B_{26}}{r}\right) w_{0} w_{0}^{\prime}+\frac{1}{r}\left(B_{16}+\frac{D_{16}}{r}\right) w_{0} w_{0}^{\prime \prime \prime}+\frac{2 B_{16}}{r} w_{0}^{\prime} w_{0}^{\prime \prime} \\
& -\frac{1}{r}\left(A_{16}-\frac{B_{16}}{r}\right) u_{0}^{\prime 2} w_{0}^{\prime}+\frac{1}{r^{2}}\left(A_{26}-\frac{B_{26}}{r}\right) u_{0}^{\prime} w_{0} w_{0}^{\prime}+\frac{1}{r}\left(B_{16}-\frac{D_{16}}{r}\right) u_{0}^{\prime} w_{0}^{\prime} w_{0}^{\prime \prime}=0, \\
& \left(\frac{B_{16}+M_{x \phi}^{*}}{r}+N_{x \phi}^{*}\right) u_{0}^{\prime}+\left(M_{x \phi}^{*}-\frac{D_{16}}{r}\right) w_{0}^{\prime \prime}+\frac{1}{r}\left(N_{x \phi}^{*}-\frac{B_{26}}{r}\right) w_{0}+\frac{M_{x \phi}^{*}}{r} \\
& +\frac{1}{r}\left(A_{16}-A_{26}-\frac{B_{26}}{r^{2}}\right) u_{0}^{\prime} w_{0}-\frac{D_{16}}{r} u_{0}^{\prime} w_{0}^{\prime \prime}-\frac{B_{16}+B_{26}-M_{x \phi}^{*}}{r} w_{0} w_{0}^{\prime \prime}+\left(A_{16}+\frac{B_{16}}{r}\right) u_{0}^{\prime 2} \\
& -\frac{A_{26}}{r^{2}} w_{0}^{2}-D_{16} w_{0}^{\prime \prime 2}+\frac{B_{26}}{r^{2}} w_{0}^{2} w_{0}^{\prime \prime}+\frac{D_{16}}{r} w_{0} w_{0}^{\prime \prime 2}-\frac{B_{16}}{r} u_{0}^{\prime} w_{0} w_{0}^{\prime \prime}=0,
\end{aligned}
$$




$$
\begin{aligned}
& B_{11} u_{0}^{\prime \prime \prime}+\left(\frac{A_{12}}{r}+p\right) u^{\prime}-D_{11} w_{0}^{i v}-\left(\frac{2 B_{12}+M_{x}^{*}-M_{\phi}^{*}}{r}+N_{x}^{*}\right) w_{0}^{\prime \prime}-\frac{1}{r}\left(\frac{A_{12}}{r}-p\right) w_{0} \\
& -\frac{B_{11}}{r} u_{0}^{\prime \prime \prime} w_{0}+\frac{B_{12}+M_{\phi}^{*}-2 B_{11}}{r} u_{0}^{\prime \prime} w_{0}^{\prime}+\left(\frac{B_{12}-B_{11}+M_{\phi}^{*}}{r}+A_{11}\right) u_{0}^{\prime} w_{0}^{\prime \prime}-\frac{p}{r} u_{0}^{\prime} w_{0} \\
& +\frac{D_{11}}{r} w_{0} w_{0}^{i v}+\frac{2 D_{11}}{r} w_{0}^{\prime} w_{0}^{\prime \prime \prime}+\frac{1}{r}\left(\frac{2 B_{12}-B_{26}}{r}-A_{12}-N_{x}^{*}\right) w_{0} w_{0}^{\prime \prime} \\
& +\left(\frac{D_{11}-D_{12}}{r}-B_{11}\right) w_{0}^{\prime \prime 2}+\frac{2 B_{12}-B_{26}}{r^{2}} w_{0}^{\prime 2}+\frac{2 B_{12}}{r} u_{0}^{\prime} u_{0}^{\prime \prime} w_{0}^{\prime}-\frac{D_{12}}{r} u_{0}^{\prime} w_{0}^{\prime} w_{0}^{\prime \prime \prime} \\
& -\frac{1}{r}\left(A_{11}+\frac{B_{26}}{r}\right) u_{0}^{\prime} w_{0} w_{0}^{\prime \prime}-\frac{D_{12}}{r} u_{0}^{\prime} w_{0}^{\prime \prime 2}-\frac{B_{26}}{r^{2}} u_{0}^{\prime} w_{0}^{\prime 2}+\frac{B_{12}}{r} u_{0}^{\prime 2} w_{0}^{\prime \prime}-\frac{B_{26}}{r^{2}} u_{0}^{\prime \prime} w_{0} w_{0}^{\prime} \\
& -\frac{D_{12}}{r} u_{0}^{\prime \prime} w_{0}^{\prime} w_{0}^{\prime \prime}+\frac{A_{12}}{r^{2}} w_{0}^{2} w_{0}^{\prime \prime}+\frac{B_{11}}{r} w_{0} w_{0}^{\prime \prime 2}+\frac{N_{\phi}^{*}}{r}=0,
\end{aligned}
$$

where ()$^{\prime}$ indicates ordinary derivative with respect to the $x$-coordinate.

As discussed, equations (34) through (37) form a system of four simultaneous nonlinear ordinary differential equations in two unknowns. This system of equations can be further reduced to a system of two simultaneous ordinary linear differential equations, through the following procedure: (i) eliminate the $w_{0} w_{0}^{\prime}$ term from equation (35) by substituting from equation (34); (ii) differentiate the new equation with respect to $x$; (iii) eliminate the $w_{0}^{\prime} w_{0}^{\prime \prime \prime}$ term from equation (37) by substituting from the new equation; and linearize the system by application of the following assumptions: (a) the in-plane normal strains, $\varepsilon_{x}^{0}$ and $\varepsilon_{\phi}^{0}$, are very small compared to unity, and (b) the out of plane rotations, $w_{0}^{\prime}$, are small. The final system of equations can be written as:

$$
\begin{aligned}
& \left(\frac{B_{16}+M_{x \phi}^{*}}{r}+N_{x \phi}^{*}\right) u_{0}^{\prime}+\left(M_{x \phi}^{*}-\frac{D_{16}}{r}\right) w_{0}^{\prime \prime}+\frac{1}{r}\left(N_{x \phi}^{*}-\frac{B_{26}}{r}\right) w_{0}+\frac{M_{x \phi}^{*}}{r}=0, \\
& \left(B_{11}+\frac{2 D_{11} \xi_{1}}{\left(\xi_{4}+\xi_{5}\right) r}\right) u_{0}^{\prime \prime \prime}+\left(\frac{A_{12}}{r}+p\right) u_{0}^{\prime}+\left(\frac{2 \xi_{2}}{\left(\xi_{4}+\xi_{5}\right) r}-1\right) D_{11} w_{0}^{i v} \\
& +\left(\frac{2 D_{11} \xi_{3}}{\left(\xi_{4}+\xi_{5}\right) r}-\frac{2 B_{12}+M_{x}^{*}-M_{\phi}^{*}}{r}-N_{x}^{*}\right) w_{0}^{\prime \prime}+\frac{1}{r}\left(\frac{A_{12}}{r}-p\right) w_{0}+\frac{N_{\phi}^{*}}{r}=0,
\end{aligned}
$$

where $\xi_{i}$ are constants that involve the material properties, shell radius, prestresses, and the shell loads. They are: 


$$
\begin{aligned}
& \xi_{1}=\left(A_{16}+\frac{B_{16}}{r}-\frac{\left(3 A_{26} r+B_{26}\right)}{\left(A_{22}-2 A_{12}\right) r} A_{11}\right), \\
& \xi_{2}=\left(\frac{\left(3 A_{26} r+B_{26}\right)}{\left(A_{22}-2 A_{12}\right) r} B_{11}-B_{16}-\frac{D_{16}}{r}\right), \\
& \xi_{3}=\frac{1}{r}\left(\frac{\left(3 A_{26} r+B_{26}\right)}{\left(A_{22}-2 A_{12}\right) r}\left(A_{12}+N_{x}^{*}-N_{\phi}^{*}+p r\right)-\left(A_{26}-2 N_{x \phi}^{*}\right)-\frac{B_{26}}{r}\right), \\
& \xi_{4}=\frac{1}{r}\left(2 B_{16}-\frac{\left(3 A_{26} r+B_{26}\right)}{\left(A_{22}-2 A_{12}\right) r}\left(B_{11}-B_{12}\right)\right), \\
& \xi_{5}=\frac{-\xi_{2}}{r} .
\end{aligned}
$$

Thus, the deflection of the shell is determined by solving equations (38) and (39).

\section{SOLUTION OF THE GOVERNING EQUATIONS}

The solution of the governing equations of a laminated cylindrical shell under constant uniform lateral pressure is obtained. Equation (38) can be rearranged such that all terms involving the radial displacement are grouped on one side of the equality while the axial displacement terms are on the other side.

$$
\left(\frac{B_{16}+M_{x \phi}^{*}}{r}+N_{x \phi}^{*}\right) u_{0}^{\prime}=\left(\frac{D_{16}}{r}-M_{x \phi}^{*}\right) w_{0}^{\prime \prime}+\frac{1}{r}\left(\frac{B_{26}}{r}-N_{x \phi}^{*}\right) w_{0}-\frac{M_{x \phi}^{*}}{r} .
$$

Next, define the constants $\alpha, \beta$, and $\gamma$ as:

$$
\begin{aligned}
& \alpha=\frac{D_{16}}{B_{16}+M_{x \phi}^{*}+N_{x \phi}^{*} r}-r \gamma, \\
& \beta=\frac{B_{26}-N_{x \phi}^{*} r}{r\left(B_{16}+M_{x \phi}^{*}+N_{x \phi}^{*} r\right)}, \\
& \gamma=\frac{M_{x \phi}^{*}}{B_{16}+M_{x \phi}^{*}+N_{x \phi}^{*} r} .
\end{aligned}
$$

Then, equation (41), its derivative and anti-derivative become:

$$
\begin{aligned}
& u_{0}^{\prime}=\alpha w_{0}^{\prime \prime}+\beta w_{0}-\gamma, \\
& u_{0}^{\prime \prime \prime}=\alpha w_{0}^{i v}+\beta w_{0}^{\prime \prime}, \\
& u_{0}=\alpha w_{0}^{\prime}+\beta \int_{x} w_{0}(\chi) d \chi-\gamma \chi+C_{5},
\end{aligned}
$$


where $C_{5}$ is the constant of integration. Substituting the results given in equation (43) into equation (39) yields a single fourth order ordinary differential equation to be solved for the radial displacement, $w_{0}$. After collecting terms, this equation becomes:

$$
\begin{aligned}
& \left(\left(B_{11}+\frac{2 D_{11} \xi_{1}}{\left(\xi_{4}+\xi_{5}\right) r}\right) \alpha+\frac{2 \xi_{2} D_{11}}{\left(\xi_{4}+\xi_{5}\right) r}-D_{11}\right) w_{0}^{i v} \\
& +\left(\left(B_{11}+\frac{2 D_{11} \xi_{1}}{\left(\xi_{4}+\xi_{5}\right) r}\right) \beta+\left(\frac{A_{12}}{r}-p\right) \alpha+\frac{2 D_{11} \xi_{3}}{\left(\xi_{4}+\xi_{5}\right) r}-\frac{2 B_{12}+M_{x}^{*}-M_{\phi}^{*}}{r}-N_{x}^{*}+P\right) w_{0}^{\prime \prime} \\
& +\frac{1}{r}\left(\frac{A_{12}}{r}-p\right)(1+\beta r) w_{0}+\frac{N_{\phi}^{*}-\left(A_{12}-p r\right) \gamma}{r}=0 .
\end{aligned}
$$

To simplify the notation in the solution of the governing differential equation for the radial displacement, introduce the following constants:

$$
\begin{aligned}
& a_{0}=\frac{1}{\left(B_{11}+\frac{2 D_{11} \xi_{1}}{\left(\xi_{4}+\xi_{5}\right) r}\right) \alpha+\frac{2 \xi_{2} D_{11}}{\left(\xi_{4}+\xi_{5}\right) r}-D_{11}}, \\
& a_{1}=\left(\begin{array}{c}
\left(B_{11}+\frac{2 D_{11} \xi_{1}}{\left(\xi_{4}+\xi_{5}\right) r}\right) \beta+\left(\frac{A_{12}}{r}-p\right) \alpha+\frac{2 D_{11} \xi_{3}}{\left(\xi_{4}+\xi_{5}\right) r} \\
-\frac{2 B_{12}+M_{x}^{*}-M_{\phi}^{*}}{r}-N_{x}^{*}
\end{array}\right) a_{0}, \\
& a_{2}=\frac{1}{r}\left(\frac{A_{12}}{r}-p\right)(1+\beta r) a_{0}, \\
& a_{3}=\frac{N_{\phi}^{*}-\left(A_{12}-p r\right) \gamma}{r} a_{0} .
\end{aligned}
$$

Then, equation (44) can be rewritten as:

$$
\frac{\mathrm{d}^{4} w_{0}}{\mathrm{~d} x^{4}}+a_{1} \frac{\mathrm{d}^{2} w_{0}}{\mathrm{~d} x^{2}}+a_{2} w_{0}+a_{3}=0
$$

This, then, is the equation to be solved for the radial displacement of a cylindrical laminated shell under constant uniform lateral and axial compression. This is a nonhomogeneous linear ordinary differential equation with constant coefficients. At this point in the solution no comments can be made on the relative magnitude of the coefficients, $a_{i}$. Therefore, the solution is: 


$$
\begin{aligned}
w_{0}(x) & =C_{1} \exp \left(\sqrt{\frac{b-a_{1}}{2} x}\right)+C_{2} \exp \left(-\sqrt{\frac{b-a_{1}}{2} x}\right) \\
& +C_{3} \exp \left(\sqrt{\frac{-b-a_{1}}{2} x}\right)+C_{4} \exp \left(-\sqrt{\frac{-b-a_{1}}{2}} x\right)-\frac{a_{3}}{a_{2}},
\end{aligned}
$$

where $a_{i}$ are defined in equation (45), $b=\sqrt{a_{1}^{2}-4 a_{2}}$, and $C_{i}$ are the constants to be determined from the boundary conditions. With $w_{0}(x)$ known from equation (43), the axial displacement can be written as:

$$
\begin{aligned}
u_{0}(x)= & \left\{\alpha \sqrt{\frac{b-a_{1}}{2}}+\beta \sqrt{\frac{2}{b-a_{1}}}\right\}\left\{C_{1} \exp \left(\sqrt{\frac{b-a_{1}}{2} x}\right)-C_{2} \exp \left(-\sqrt{\frac{b-a_{1}}{2} x}\right)\right\} \\
& +\left\{\alpha \sqrt{\frac{-b-a_{1}}{2}}+\beta \sqrt{\frac{2}{-b-a_{1}}}\right\}\left\{C _ { 3 } \operatorname { e x p } \left(\sqrt{\left.\left.\frac{-b-a_{1}}{2} x\right)-C_{4} \exp \left(-\sqrt{\frac{-b-a_{1}}{2}} x\right)\right\}}\right.\right. \\
& \left(\gamma+\beta \frac{a_{3}}{a_{2}}\right) x+C_{5} .
\end{aligned}
$$

The boundary conditions for the shell may be defined in general by a system of springs that define the stiffness of the boundary. Figure 4 represents a schematic drawing of the proposed elastic foundation for the shell. Note that the foundation shown includes linear and torsional springs. The constants $k_{0 z}, k_{0 x} k_{L z}$, and $k_{L x}$ represent the linear stiffness of the boundaries in the $z$ - and $x$-directions, at $x=0$ and $x=L$, respectively. Further, the constants $R_{0}$ and $R_{L}$ represent the rotational stiffness of the boundaries at $x=0$ and $x=L$, respectively. The boundary conditions are: 
(1) $\quad k_{0 z} w_{0}(0)=\left.\bar{N}_{x z}\right|_{x=0}-\left.\left.\frac{1}{r} \frac{\partial \bar{M}_{x \phi}}{\partial \phi} \Rightarrow \bar{N}_{x z}\right|_{x=0} \Rightarrow \frac{\mathrm{d} \bar{M}_{x}}{\mathrm{~d} x}\right|_{x=0}$

$$
\begin{aligned}
& \left.\Rightarrow \frac{\mathrm{d} M_{x}^{o}}{\mathrm{~d} x}\right|_{x=0}\left(1-\frac{w_{0}(0)}{r}\right)-\left.\frac{M_{x}^{o}+M_{x}^{*}}{r}\right|_{x=0} w_{0}^{\prime}(0) \\
& \left.\Rightarrow \frac{\mathrm{d} M_{x}^{o}}{\mathrm{~d} x}\right|_{x=0}-\left.\frac{M_{x}^{*}}{r}\right|_{x=0} w_{0}^{\prime}(0),
\end{aligned}
$$

(2) $\quad R_{0} w_{0}^{\prime}(0)=\left.\left.\bar{M}_{x}\right|_{x=0} \Rightarrow\left(M_{x}^{o}+M_{x}^{*}\right)\right|_{x=0}\left(1-\frac{w_{0}(0)}{r}\right)$

$$
\left.\Rightarrow M_{x}^{o}\right|_{x=0}+M_{x}^{*}\left(1-\frac{w_{0}(0)}{r}\right)
$$

(3) $\quad k_{L z} w_{0}(L)=\left.\left.\bar{N}_{x z}\right|_{x=L} \Rightarrow \frac{\mathrm{d} M_{x}}{\mathrm{~d} x}\right|_{x=L}-\left.\frac{M_{x}^{*}}{r}\right|_{x=L} w_{0}^{\prime}(L)$,

(4) $\quad R_{L} w_{0}^{\prime}(0)=\left.\left.\bar{M}_{x}\right|_{x=L} \Rightarrow\left(M_{x}^{o}+M_{x}^{*}\right)\right|_{x=L}\left(1-\frac{w_{0}(L)}{r}\right)$

$$
\left.\Rightarrow M_{x}^{o}\right|_{x=L}+M_{x}^{*}\left(1-\frac{w_{0}(L)}{r}\right)
$$

(5) $\quad k_{0 x} u_{0}(0)=\left.\bar{N}_{x}\right|_{x=0} \Rightarrow\left(\left.N_{x}^{o}\right|_{x=0}+N_{x}^{*}\right)\left(1-\frac{w_{0}(0)}{r}\right)$

$$
\left.\Rightarrow N_{x}^{o}\right|_{x=0}+\left(N_{x}^{*}\right)\left(1-\frac{w_{0}(0)}{r}\right),
$$

(6) $\quad k_{L x} u_{0}(0)=\left.\bar{N}_{x}\right|_{x=L} \Rightarrow\left(\left.N_{x}^{o}\right|_{x=L}+N_{x}^{*}\right)\left(1-\frac{w_{0}(L)}{r}\right)$,

\section{where}

$$
\begin{aligned}
& M_{x}^{o}(x)=\left(B_{11} \alpha-D_{11}\right) w_{0}^{\prime \prime}(x)+\left(B_{11} \beta-\frac{B_{12}}{r}\right) w_{0}(x)-B_{11} \gamma, \\
& N_{x}^{o}(x)=\left(A_{11} \alpha-B_{11}\right) w_{0}^{\prime \prime}(x)+\left(A_{11} \beta-\frac{A_{12}}{r}\right) w_{0}(x)-A_{11} \gamma .
\end{aligned}
$$

These equations may now be expressed in matrix form such that: 
$\left[\begin{array}{lllll}k_{11} & k_{12} & k_{13} & k_{14} & k_{15} \\ k_{21} & k_{22} & k_{23} & k_{24} & k_{25} \\ k_{31} & k_{32} & k_{33} & k_{34} & k_{35} \\ k_{41} & k_{42} & k_{43} & k_{44} & k_{45} \\ k_{51} & k_{52} & k_{53} & k_{54} & k_{55}\end{array}\right]\left\{\begin{array}{l}C_{1} \\ C_{2} \\ C_{3} \\ C_{4} \\ C_{5}\end{array}\right\}=\left\{\begin{array}{l}f_{1} \\ f_{2} \\ f_{3} \\ f_{4} \\ f_{5}\end{array}\right\}$,

where $k_{i j}$ and $f_{i}$ are constants, which are complex expressions involving the material constants, radius of the shell, applied loads, and preloads, and $C_{i}$ are the constants to be determined. The expressions for $k_{i j}$ and $f_{i}$ are given in the appendix.

\section{Special Cases of the Solution Obtained}

\section{Special construction techniques}

If the laminated cylindrical shell is constructed such that the laminate is:

(1) symmetric, then the coupling stiffnesses are zero, i.e.: $B_{i j}=0, i, j=1,2,6$; and

(2) balanced, then the 1-6 and 2-6 axial stiffnesses are zero, i.e.: $A_{16}=A_{26}=0$. For this specific design the constants $\xi_{i}$ in equation (40), $\alpha, \beta$, and $\gamma$ in equation (42), and $a_{i}$ in equation (45) are greatly simplified. For this case they become:

$$
\left.\begin{array}{l}
\xi_{1}=0, \\
\xi_{2}=-\frac{D_{16}}{r}, \\
\xi_{3}=\frac{2 N_{x \phi}^{*}}{r}, \\
\xi_{4}=0, \\
\xi_{5}=\frac{-\xi_{2}}{r}, \\
\alpha=\frac{D_{16}}{M_{x \phi}^{*}+N_{x \phi}^{*} r}-r \gamma, \\
\beta=\frac{-N_{x \phi}^{*}}{M_{x \phi}^{*}+N_{x \phi}^{*} r}, \\
\gamma=\frac{M_{x \phi}^{*}}{M_{x \phi}^{*}+N_{x \phi}^{*} r},
\end{array}\right\}
$$

The solution then follows the previous pattern. 


\section{NUMERICAL CASE STUDIES}

The solution obtained was evaluated for various materials, stacking sequences, prestresses, and boundary and loading conditions. A sample of the problems addressed are shown in this section. Material property values for the solutions presented here are listed in Table 1.

Table 1. Material Property Values Used in Examples.

\begin{tabular}{|c|c|c|c|c|}
\hline Material & $\begin{array}{c}\mathrm{E}_{1} \\
\mathrm{Mpsi}\end{array}$ & $\begin{array}{c}\mathrm{E}_{2} \\
\mathrm{Mpsi}\end{array}$ & $v_{12}$ & $\begin{array}{c}\mathrm{G}_{12} \\
\mathrm{Mpsi}\end{array}$ \\
\hline E-glass/Epoxy & 5.6 & 1.2 & 0.26 & 0.6 \\
\hline IM6/Epoxy & 29.46 & 1.63 & 0.32 & 1.22 \\
\hline Kevlar-49/Epoxy & 11.03 & 0.8 & 0.34 & 0.33 \\
\hline
\end{tabular}

\section{Effect of Pre-stress}

The effect of pre-stress is shown in the analysis of a symmetric laminated composite shell of length 30 inches and diameter 10 inches. The shell in this example has twelve ply of IM6/Epoxy with a stacking sequence of $[2[ \pm 45] / 2[90]]_{2 \mathrm{~S}}$. The shell is simply supported with lateral pressure of 3000 psi. A plot of the deformed midplane location for two different pre-stress values is shown in Fig. 5. Note that in this figure the maximum radial displacement for a pre-stress of $1.78 \mathrm{lb}$./tow is of the order of magnitude of $10^{-6}$, while the maximum radial displacement for the pre-stress of $0.178 \mathrm{lb} . / \mathrm{tow}$ is of the order of magnitude of $10^{-7}$. In neither case was the internal pressure sufficient to overcome the drawing in of the shell due to the pre-stress.

\section{Effect of Lateral Pressure with Constant Pre-stress}

The solution obtained was evaluated for a simply-supported 33 ply hybrid composite shells of length 10 and 5 inches and diameter 10 inches. The stacking sequence used for the hybrid laminate is asymmetric and is $\left[2[ \pm 45]_{\text {E-glass/ Epoxy }} /[4[90 / \pm 45 / 0 / \pm 45] / 90]_{\mathrm{IM} 6 / \text { Epoxy }} / 2[ \pm 45]_{\text {Kevlar-49/Epoxy }}\right]_{\mathrm{T}}$. The position of the deformed shell midplane is shown in Figs. 6 and 7. Fig. 6 shows the effect of lateral pressure for constant pre-stress. The pre-stress for all examples in Figs. 6 and 7 is $0.375 \mathrm{lbs}$./tow; the width per tow is 1 inch and the tow diameter is 0.005 inches. The effect of the length on the deformed geometry is shown in Fig. 7 for a lateral pressure of 3000 psi.

The results shown in Fig. 6 are consistent with those shown by Chaudhuri, Balaraman, and Kunukkasseril ${ }^{1}$ for an anisotropic cylindrical shell under internal pressure. Note that the deflection increases (for the coordinate system used, negative deflection is away from the centerline of the shell) as lateral pressure increases. The plot shows that the deformed length decreases as lateral pressure increase, as expected with simple supports. 


\section{CONCLUSION}

A new closed form solution for cylindrical shells has been developed. Features of the solution address nonlinear effects due to large deformations, material orthotropy, and effects of pre-stress due to a winding process. Equations are presented to evaluate the solution with flexible or elastic boundary conditions. The solution allows analysis of shells with stacking sequences that are not symmetric.

Results from the numerical examples presented indicate that: an order of magnitude increase in the winding pre-stress can result in an order of magnitude increase in the shell deformation; and the deformed shape of the shell changes dramatically as the length to diameter ratio changes. Results are presented for symmetric and asymmetric laminates.

\section{REFERENCES}

[1] Jones, R. M., Mechanics of Composite Materials, McGraw-Hill, New York, 1975.

[2] Tsai, S. W., and Hahn, H. J., Introduction to Composite Materials, Technomics, Westport, CT, 1969.

[3] Ashton, J. E., Halpin, J. C. , and Petit, P. H., Primer of Composite Materials: Analysis, Technomics, Westport, CT, 1969.

[4] Timoshenko, S., and Woinowsky-Krieger, S., Theory of Plates and Shells, Second Edition, McGraw-Hill, New York, 1959.

[5] Timoshenko, S., and Gere, J. M., Theory of Elastic Stability, Second Edition, McGraw-Hill, New York, 1959.

[6] Chaudhuri, R. A., Balaraman, K., and Kunukkasseril V. X., "Arbitrarily Laminated, Anisotropic Cylindrical Shell Under Internal Pressure," AIAA J., Vol. 24, No. 11, November, 1986.

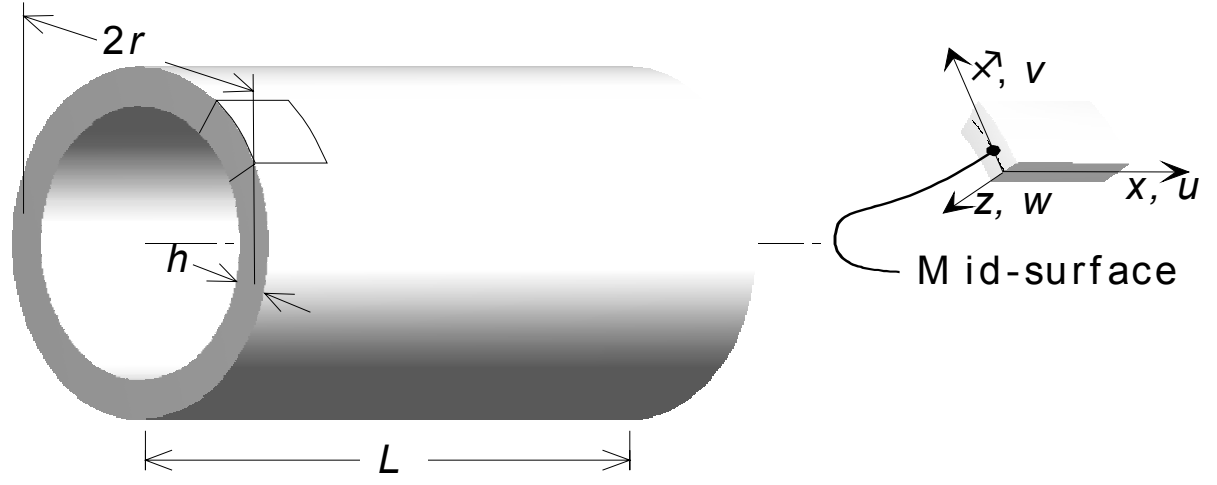

Fig.1. Axisymmetric Shell Dimensions and Associated Coordinate System with Displacement Directions. 


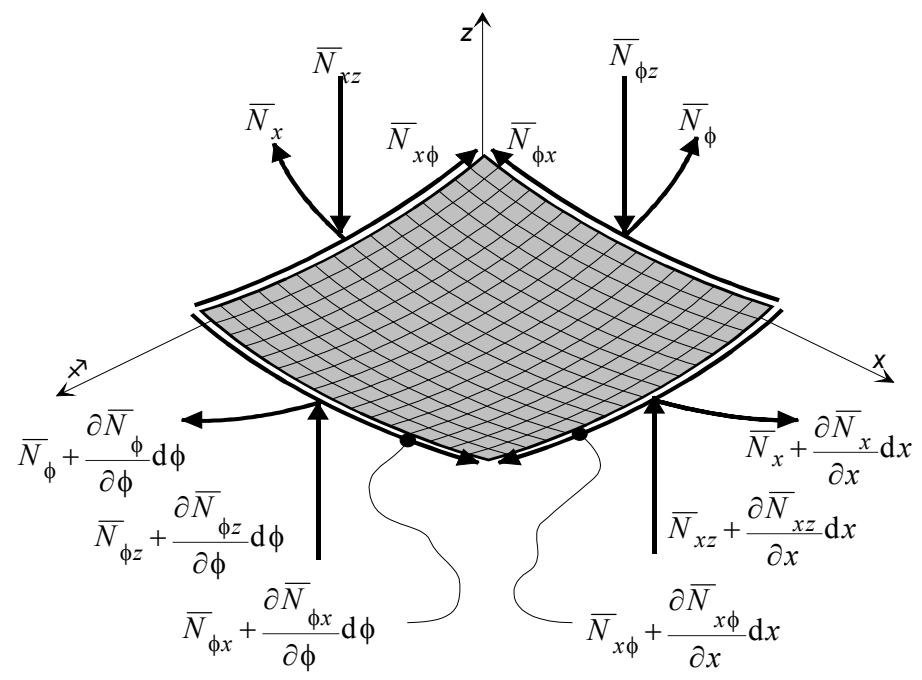

Fig. 2. Force Resultants on the Midplane of an Infinitesmal Shell Element in the Deformed Configuration.

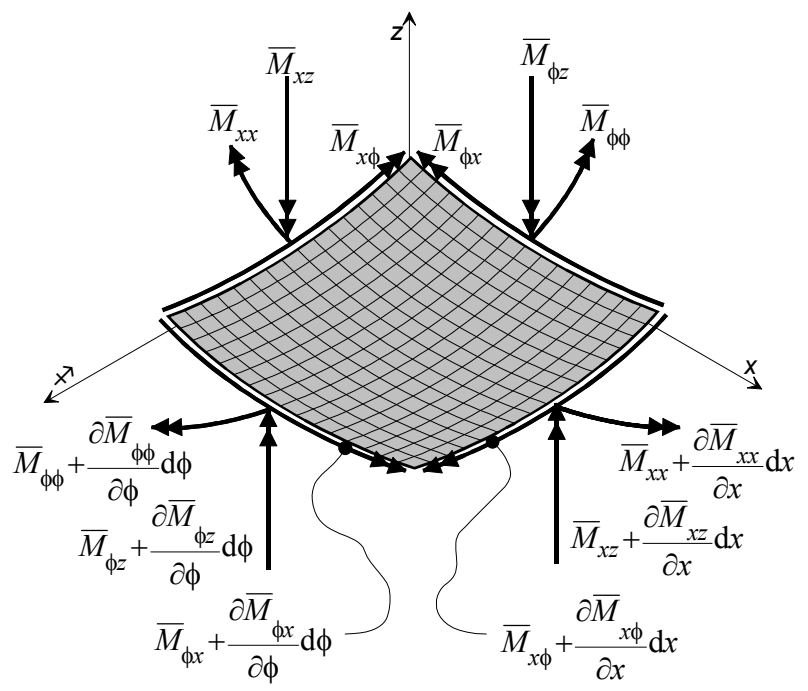

Fig. 3. Moment Resultants on the Midplane of an Infinitesmal Shell Element in the Deformed Configuration.

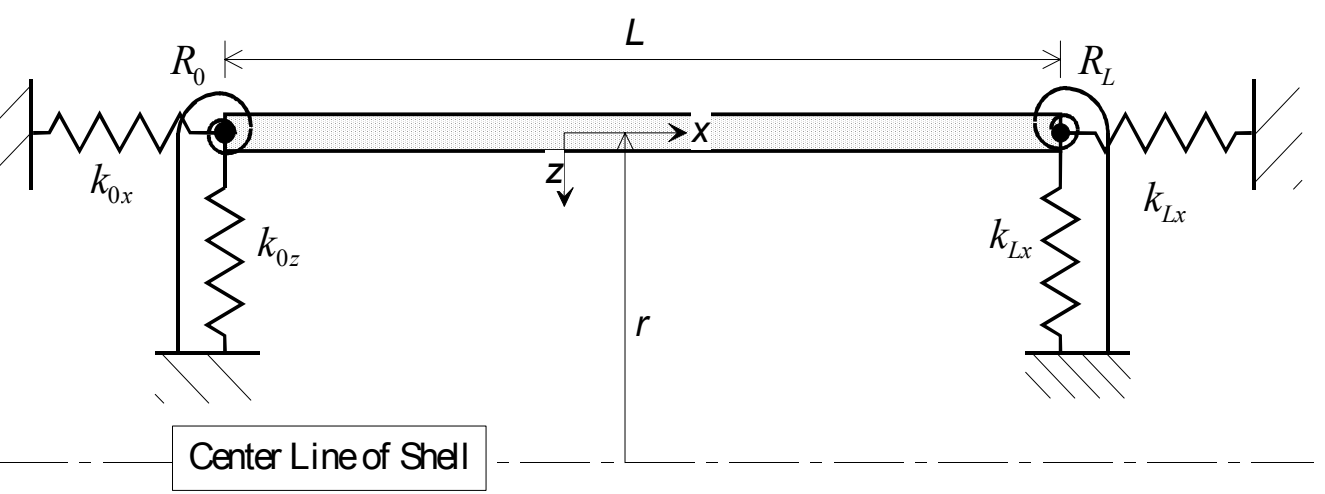

Fig. 4. Shell Boundary Conditions 


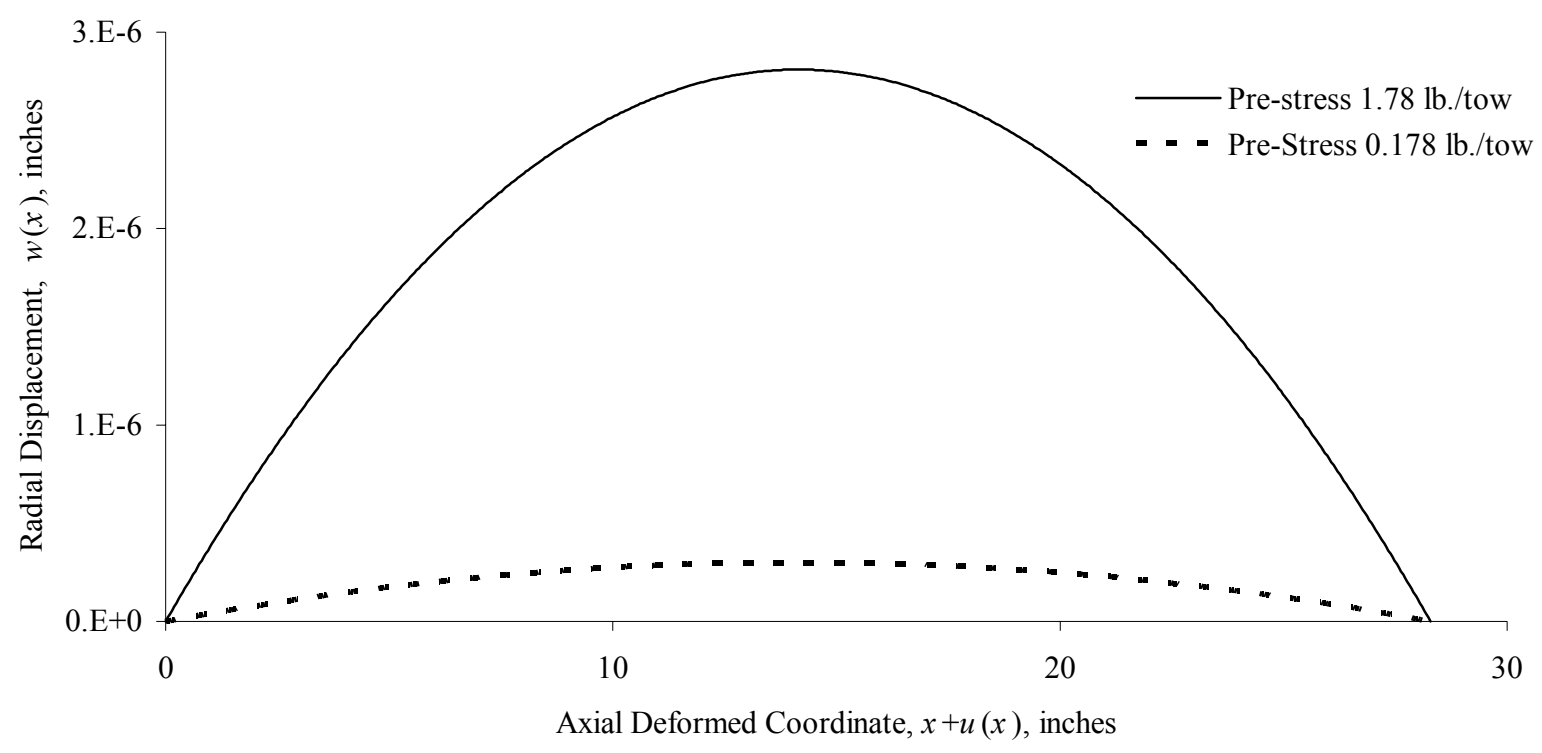

Fig. 5. Plot of Deformed Midplane Location Showing Effect of Pre-Stress With Constant Loading Conditions. 
Axial Deformed Coordinate, $x+u(x)$, inches

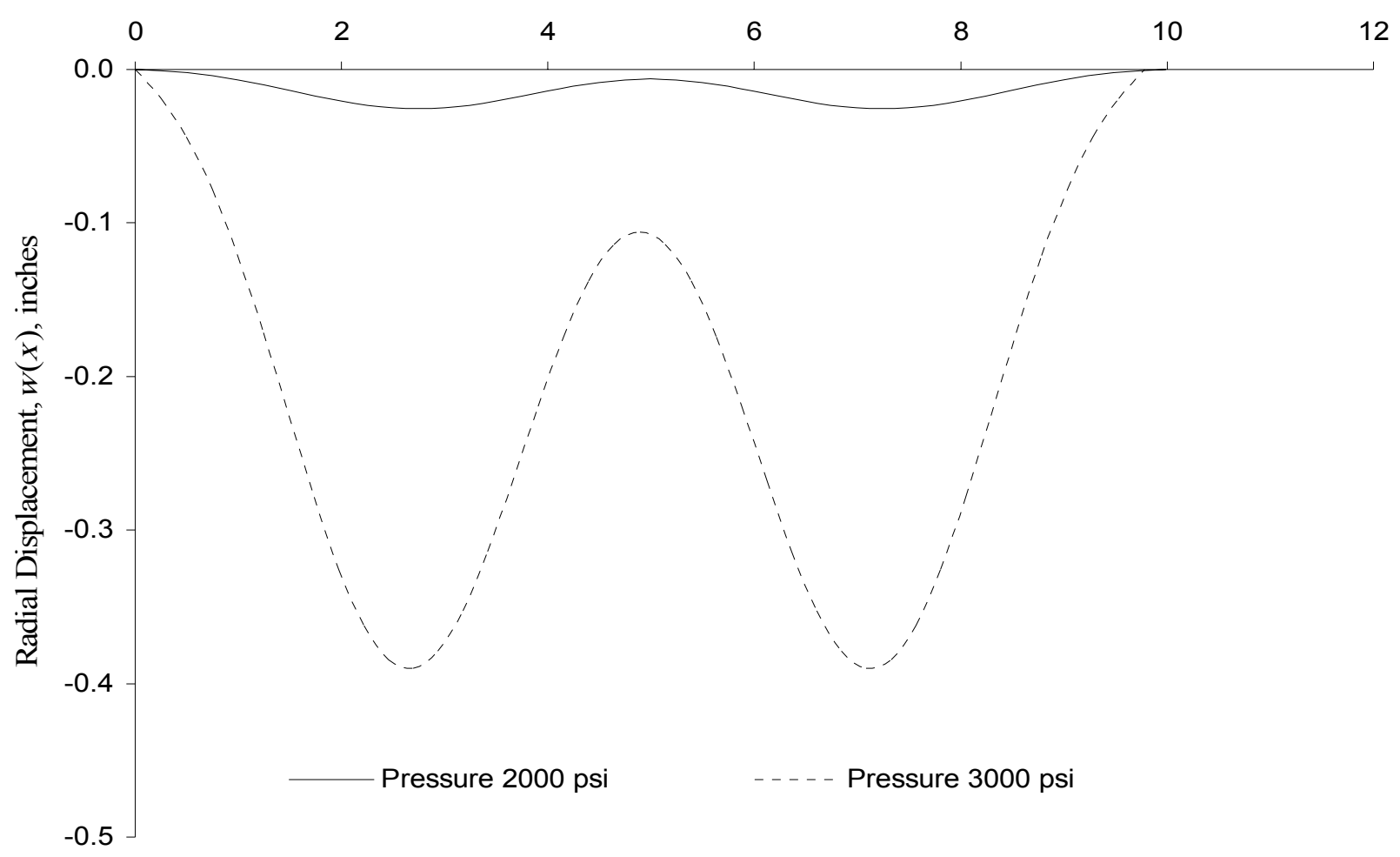

Fig. 6. Deformed Midplane of Hybrid Laminated Cylindrical Shell for Various Loading Conditions with Simple Supports and a Constant Pre-Stress of 0.375 lbs./tow.

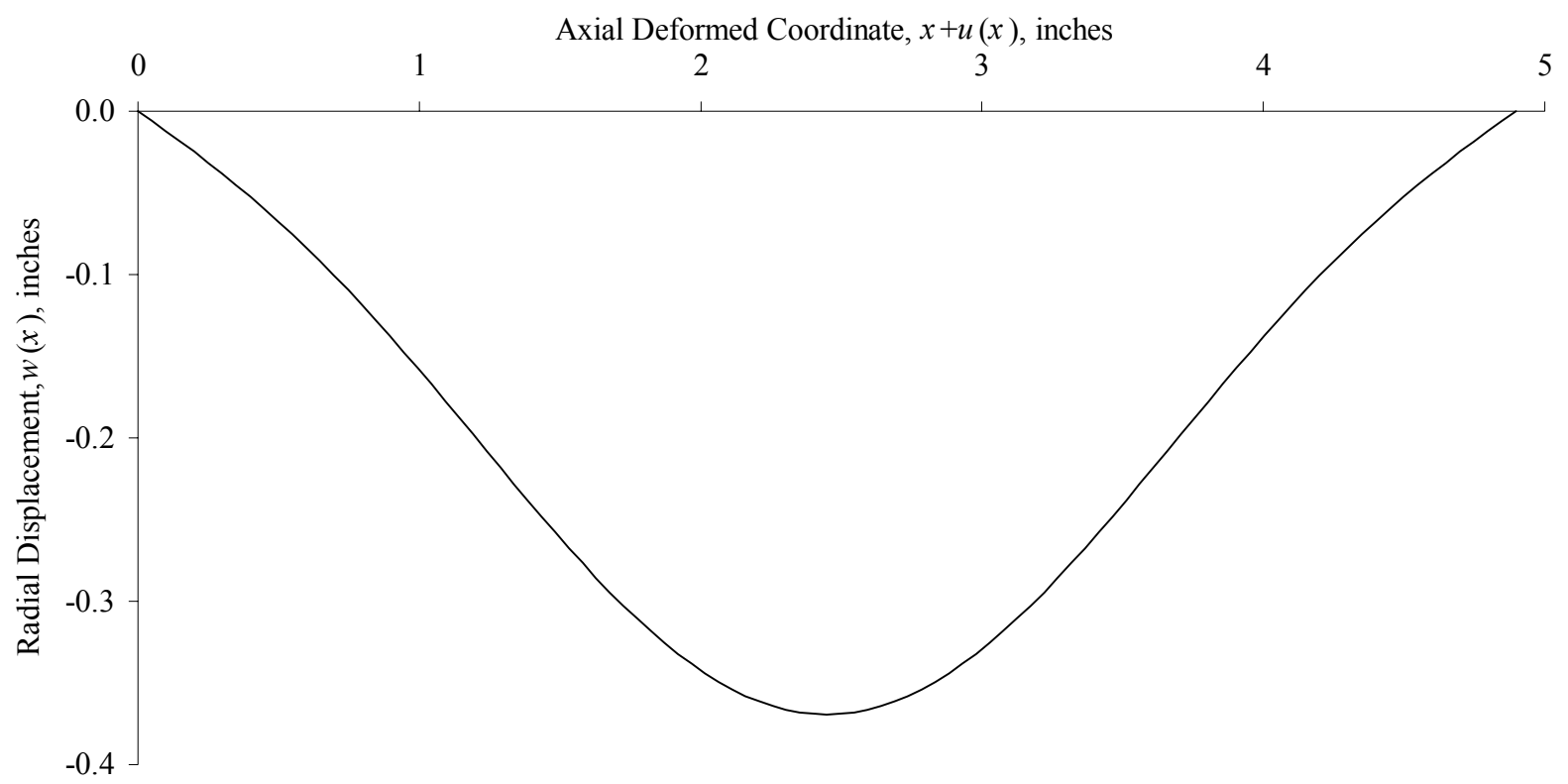

Fig. 7. Deformed Midplane of Hybrid Laminated Cylindrical Shell for Length of 5 Inches, Simply Supported, and Lateral Pressure of 3000 psi. 


\section{APPENDIX}

The coefficients of the matrix equation (50) $k_{i j}, f_{j}$ will take the form :

$$
\begin{aligned}
& k_{11}=k_{0 z}-\Omega_{1}+\frac{M_{X}^{*}}{r} \sqrt{\frac{b-a_{1}}{2}}, \\
& k_{12}=k_{0 z}+\Omega_{1}-\frac{M_{x}^{*}}{r} \sqrt{\frac{b-a_{1}}{2}}, \\
& k_{13}=k_{0 z}-\Omega_{2}+\frac{M_{x}^{*}}{r} \sqrt{\frac{-\left(b+a_{1}\right)}{2}} \text {, } \\
& k_{14}=k_{0 z}+\Omega_{2}-\frac{M_{x}^{*}}{r} \sqrt{\frac{-\left(b+a_{1}\right)}{2}} \text {, } \\
& k_{21}=R_{0} \sqrt{\frac{b-a_{1}}{2}}-\Omega_{3}+\frac{M_{x}^{*}}{r}, \\
& k_{22}=-R_{0} \sqrt{\frac{b-a_{1}}{2}}-\Omega_{3}+\frac{M_{x}^{*}}{r}, \\
& k_{23}=R_{0} \sqrt{\frac{-\left(b+a_{1}\right)}{2}}+\Omega_{4}+\frac{M_{x}^{*}}{r}, \\
& k_{24}=-R_{0} \sqrt{\frac{-\left(b+a_{1}\right)}{2}}+\Omega_{4}+\frac{M_{x}^{*}}{r}, \\
& k_{31}=\left(k_{L z}-\Omega_{1}+\frac{M_{x}^{*}}{r} \sqrt{\frac{b-a_{1}}{2}}\right) \exp \left(\sqrt{\frac{b-a_{1}}{2}} L\right), \\
& k_{32}=\left(k_{L z}+\Omega_{1}-\frac{M_{x}^{*}}{r} \sqrt{\frac{b-a_{1}}{2}}\right) \exp \left(-\sqrt{\frac{b-a_{1}}{2}} L\right), \\
& k_{33}=\left(k_{L z}-\Omega_{2}+\frac{M_{x}^{*}}{r} \sqrt{\frac{-\left(b+a_{1}\right)}{2}}\right) \exp \left(\sqrt{\frac{-\left(b+a_{1}\right)}{2}} L\right), \\
& k_{34}=\left(k_{L z}+\Omega_{2}-\frac{M_{x}^{*}}{r} \sqrt{\frac{-\left(b+a_{1}\right)}{2}}\right) \exp \left(-\sqrt{\frac{-\left(b+a_{1}\right)}{2}} L\right), \\
& k_{41}=\left(R_{L} \sqrt{\frac{b-a_{1}}{2}}-\Omega_{3}+\frac{M_{x}^{*}}{r}\right) \exp \left(\sqrt{\frac{b-a_{1}}{2}} L\right),
\end{aligned}
$$




$$
\begin{aligned}
& k_{42}=\left(-R_{L} \sqrt{\frac{b-a_{1}}{2}}-\Omega_{3}+\frac{M_{x}^{*}}{r}\right) \exp \left(-\sqrt{\frac{b-a_{1}}{2}} L\right), \\
& k_{43}=\left(R_{L} \sqrt{\frac{-\left(b+a_{1}\right)}{2}}+\Omega_{4}+\frac{M_{x}^{*}}{r}\right) \exp \left(\sqrt{\frac{-\left(b+a_{1}\right)}{2}} L\right), \\
& k_{44}=\left(-R_{L} \sqrt{\frac{-\left(b+a_{1}\right)}{2}}+\Omega_{4}+\frac{M_{x}^{*}}{r}\right) \exp \left(-\sqrt{\frac{-\left(b+a_{1}\right)}{2}} L\right) \\
& k_{15}=k_{25}=k_{35}=k_{45}=0 \text {, } \\
& k_{51}=k_{0 x}\left(\alpha \sqrt{\frac{b-a_{1}}{2}}+\beta \sqrt{\frac{2}{b-a_{1}}}\right)+\Omega_{5}, \\
& k_{52}=-k_{0 x}\left(\alpha \sqrt{\frac{b-a_{1}}{2}}+\beta \sqrt{\frac{2}{b-a_{1}}}\right)+\Omega_{5} \text {, } \\
& k_{53}=k_{0 x}\left(\alpha \sqrt{\frac{-\left(b+a_{1}\right)}{2}}+\beta \sqrt{\frac{-2}{b+a_{1}}}\right)+\Omega_{6}, \\
& k_{54}=-k_{0 x}\left(\alpha \sqrt{\frac{-\left(b+a_{1}\right)}{2}}+\beta \sqrt{\frac{-2}{b+a_{1}}}\right)+\Omega_{6}, \\
& k_{55}=k_{0 x} \quad,
\end{aligned}
$$

and

$$
\begin{aligned}
f_{1}=k_{0 z}\left(\frac{a_{3}}{a_{2}}\right), \\
f_{2}=\left(\frac{M_{x}^{*}-B_{11} \beta r+B_{12}}{r}\right)\left(\frac{a_{3}}{a_{2}}\right)+M_{x}^{*}-B_{11} \gamma, \\
f_{3}=k_{L z}\left(\frac{a_{3}}{a_{2}}\right), \\
f_{4}=\left(\frac{M_{x}^{*}-B_{11} \beta r+B_{12}}{r}\right)\left(\frac{a_{3}}{a_{2}}\right)+M_{x}^{*}-B_{11} \gamma, \\
f_{5}=\left(\frac{M_{x}^{*}+A_{12}-A_{11} \beta r}{r}\right)\left(\frac{a_{3}}{a_{2}}\right)+N_{x}^{*}-A_{11} \gamma,
\end{aligned}
$$

where, 


$$
\begin{aligned}
& \Omega_{1}=\left(B_{11} \alpha-D_{11}\right)\left(\frac{b-a_{1}}{2}\right)^{\frac{3}{2}}+\left(\frac{B_{11} \beta r-B_{12}}{r}\right)\left(\frac{b-a_{1}}{2}\right)^{\frac{1}{2}}, \\
& \Omega_{2}=\left(B_{11} \alpha-D_{11}\right)\left(\frac{-b-a_{1}}{2}\right)^{\frac{3}{2}}+\left(\frac{B_{11} \beta r-B_{12}}{r}\right)\left(\frac{-b-a_{1}}{2}\right)^{\frac{1}{2}}, \\
& \Omega_{3}=\left(B_{11} \alpha-D_{11}\right)\left(\frac{b-a_{1}}{2}\right)+\left(\frac{B_{11} \beta r-B_{12}}{r}\right), \\
& \Omega_{4}=\left(B_{11} \alpha-D_{11}\right)\left(\frac{b+a_{1}}{2}\right)-\left(\frac{B_{11} \beta r-B_{12}}{r}\right), \\
& \Omega_{5}=N_{x}^{*}-\left(A_{11} \alpha-B_{11}\right)\left(\frac{b-a_{1}}{2}\right)-\left(\frac{A_{11} \beta r-A_{12}}{r}\right), \\
& \Omega_{6}=N_{x}^{*}+\left(A_{11} \alpha-B_{11}\right)\left(\frac{b+a_{1}}{2}\right)-\left(\frac{A_{11} \beta r-A_{12}}{r}\right) .
\end{aligned}
$$

